\title{
Bietti's tapetoretinal degeneration with marginal corneal dystrophy (crystalline retinopathy): case report
}

\author{
ROSALIND J HARRISON,' R RONALD ACHESON, \\ AND J CHRISTOPHER DEAN-HART ${ }^{1}$ \\ From the ${ }^{1}$ Bristol Eye Hospital, Bristol, and ${ }^{2}$ Taunton and Somerset Hospital, Taunton
}

SUMMARY A patient with Bietti's tapetoretinal degeneration and marginal corneal dystrophy is reported on. Refractile deposits found in both the retina and cornea are the most striking feature of this condition. Apart from a slight reduction in left visual acuity there were no visual symptoms. The dark adapted electroretinogram was abnormal. No metabolic disturbance was detected.

A tapetoretinal degeneration combined with a marginal corneal dystrophy was described by Bietti in three patients (two of whom were brothers) in 1937. ${ }^{1}$ The fundus picture was reported to be similar to that of retinitis punctata albescens, but the spots, located mainly at the posterior pole, were refractile, polygonal, and could be seen at all levels of the neuroretina. Pigment changes were seen at the fovea and the retinal periphery. The corneal deposits were numerous, very small, yellowish white, and concentrated in the superficial layers of the cornea near the limbus. Eight similar cases have since been reported, ${ }^{23}$ and the term crystalline retinopathy has been applied to the fundus appearance. ${ }^{3}$ Our case is the first to be reported in Britain.

\section{Case history}

A 23-year-old Caucasian woman attended her optician for a routine refraction. She had no visual symptoms and the visual acuity was recorded as $6 / 5$ in each eye. Some pigmentary changes were noted at both maculae, and on referral to an ophthalmologist the retinal findings were confirmed, though no further action was taken. Six years later she became aware of slight reduction in her left visual acuity and was reassessed.

Her history was unremarkable. She was the only child of unrelated parents and there was no family history of any ocular disorder. Apart from allergic Correspondence to Miss R Harrison, FRCS, Burton District Hospital Centre, Belvedere Road, Burton-on-Trent. rhinitis her health was excellent. Dietary habits were normal and she was not taking any regular medication. There was no history of night blindness.

On examination her visual acuities were $6 / 5$ right and $6 / 6$ left unaided. Fine dot-like opacities were present in the superficial layers of the corneal stroma at $360^{\circ}$ of the limbus in both eyes. These opacities had the appearance of tiny crystals (Fig. 1). The anterior segments were otherwise normal. Examination of the fundi showed pigment clumping at both maculae, more marked on the right than on the left. Yellowish white crystalline deposits were scattered over the posterior poles of both eyes. They were detected in all layers of the neuroretina and some lay in front of blood vessels (Fig. 2, 3). Small clumps of retinal pigment extended to the equator, but the retina beyond the equator appeared normal (Fig. 4). Visual fields were full (Friedman field analyser and Goldmann perimeter), and colour vision was normal (100 hue test).

Fluorescein angiography showed atrophy of the retinal pigment epithelium at both posterior poles, with an intact choriocapillaris and normal retinal vasculature. The crystalline deposits did not fluoresce (Fig. 5).

Electrodiagnostic tests revealed normal ' $a$ ' and ' $b$ ' waves of the electroretinogram (ERG) of both eyes during light adaptation in terms of both latency and amplitude. The electro-oculograms (EOGs) were also normal in both eyes. The ERG of both eyes and the electroencephalogram (EEG) followed flickering light up to $64 \mathrm{~Hz}$. The ' $b$ ' scotopic wave of the ERG of 


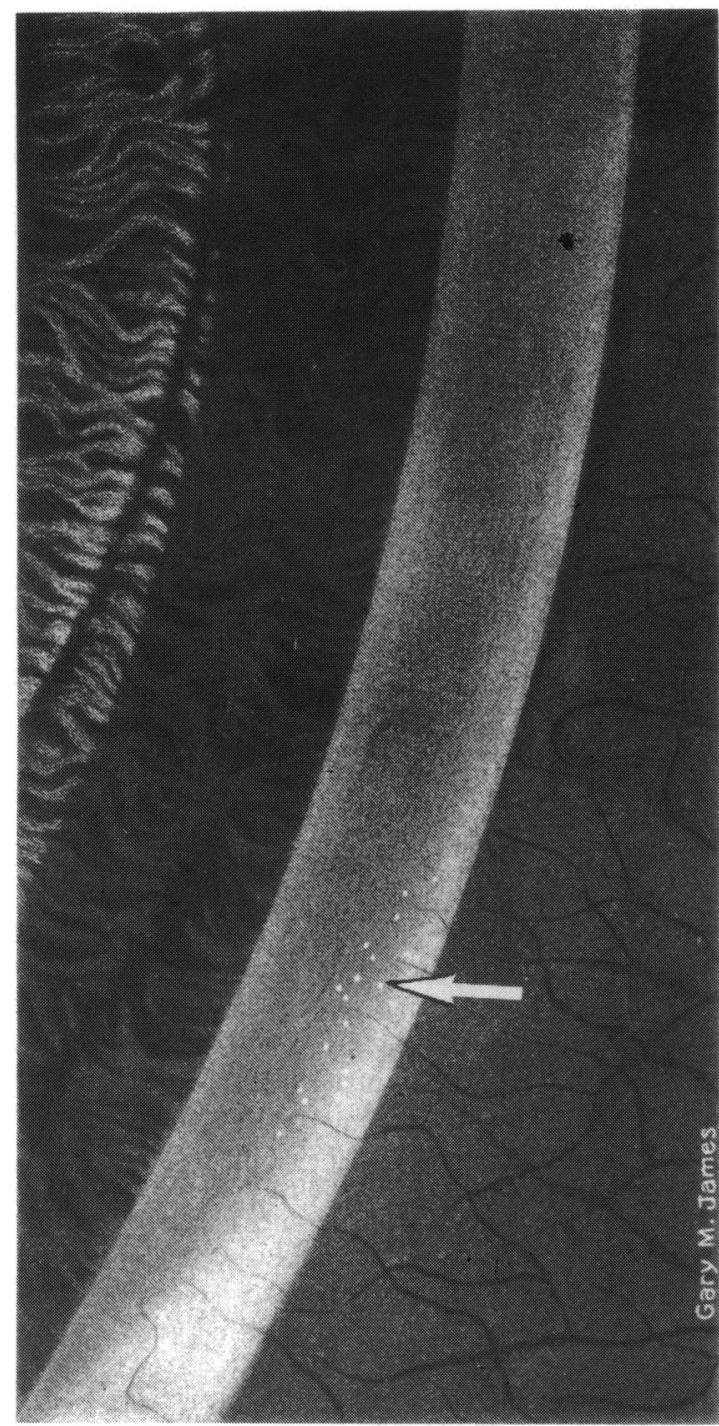

Fig. 1 Artist's drawing of the corneal deposits (arrowed).

both eyes, following stimulation with blue flashes, did not develop after 10 minutes of dark adaptation and achieved very small values during the following 20 minutes. These findings indicate that the scotopic responses were impaired. Visually evoked pattern reversal responses were normal.

A search of an underlying metabolic disorder was unrewarding, as the results of all the following investigations were normal: plasma urea, electrolytes, and creatinine; plasma and urine amino acid screens; fasting plasma triglyceride and cholesterol; quantitative lipoprotein assays. The 24-hour urine oxalate excretion was $0 \cdot 12 \mathrm{mmol}$, which is low.

A number of lysosomal enzymes involved in

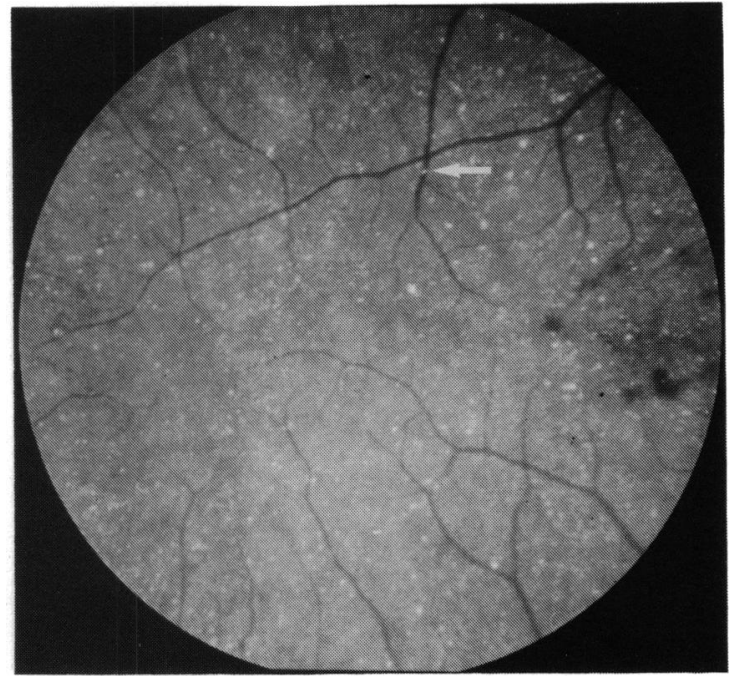

Fig. 2 Fundus photograph of the right eye, showing macular pigmentary changes and numerous crystalline deposits, some lying in front of blood vessels (arrowed).

glycolipid and oligosaccharide metabolism were assayed in leucocytes and in cultured skin fibroblasts. These were $\beta$-galactosidase, hexosaminidase $A$ and $B, \beta$-glucuronidase, $\alpha$-fucosidase, $\alpha$-mannosidase, and aryl sulphatase. No abnormality was found, indicating that Bietti's disease is probably not a previously unrecognised lysosomal storage disorder.

\section{Discussion}

The clinical picture of a marginal corneal dystrophy and crystalline retinopathy provides little latitude with regard to the differential diagnosis, as few conditions show refractile bodies in both cornea and retina. The crystals of cystinosis are found throughout the entire cornea, not solely at the limbus, and appear in the choroid and retinal pigment epithelium but not the neuroretina. ${ }^{4}$ Corneal abnormalities have not been noted in cases of crystalline retinopathy of known cause, but they may have been overlooked. White retinal flecks, confirmed as oxalate crystals at necropsy, have been seen in a few patients with primary oxalosis ${ }^{5}$ and in a patient with oxalosis secondary to prolonged methoxyfluorane anaesthesia. ${ }^{6}$ There was no evidence of primary or secondary oxalosis in our patient. Refractile bodies may be found in the retina in cases of gyrate atrophy, the Sjögren-Larsson syndrome, ${ }^{8}$ and in patients treated with tamoxifen, ${ }^{9}$ but the underlying condition is evident from the clinical history and examination.

The nature of the refractile deposits and any underlying metabolic abnormality in Bietti's disease remains unknown. A patient with hypobetalipopro- 


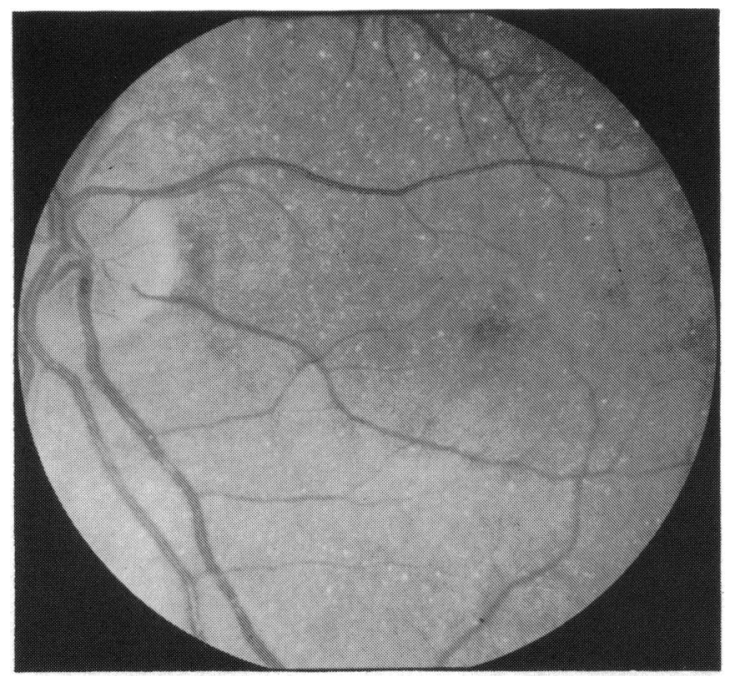

Fig. 3 Fundus photograph of the left eye, similar to the right but with less obvious macular pigmentary changes.

teinaemia had a fundus picture said to resemble a crystalline retinopathy, ${ }^{10}$ but three patients with Bietti's disease had raised serum cholesterol ${ }^{12}$ (it is low in hypobetalipoproteinaemia) and the remainder had normal serum lipids and lipoproteins. ${ }^{23}$ Two patients underwent corneal and conjunctival biopsy. Lipid inclusion bodies were found in the corneal and conjunctival fibroblasts and in a few corneal epithelial cells in one case, but no refractile deposits were seen. ${ }^{3}$ The other biopsy was reported as normal. $^{2}$

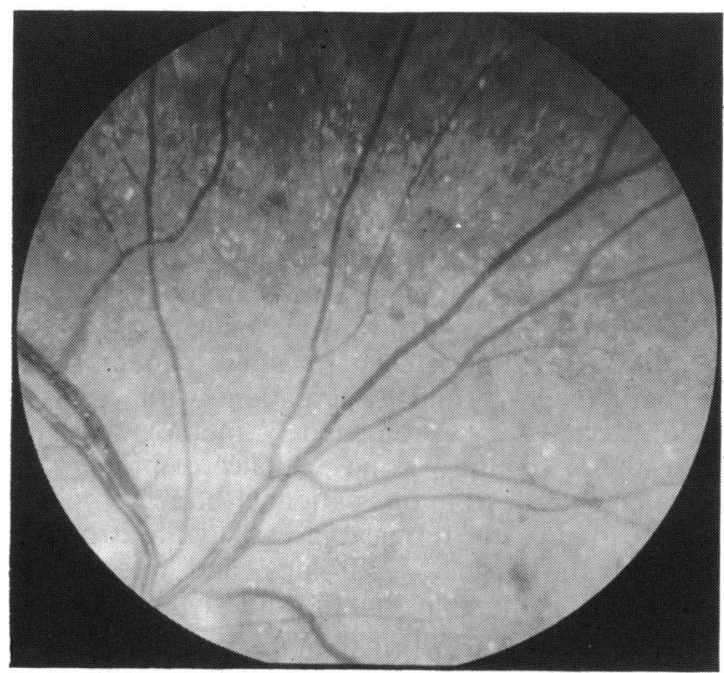

Fig. 4 Fundus photograph of the right eye, showing crystalline deposits and peripheral pigmentary changes.

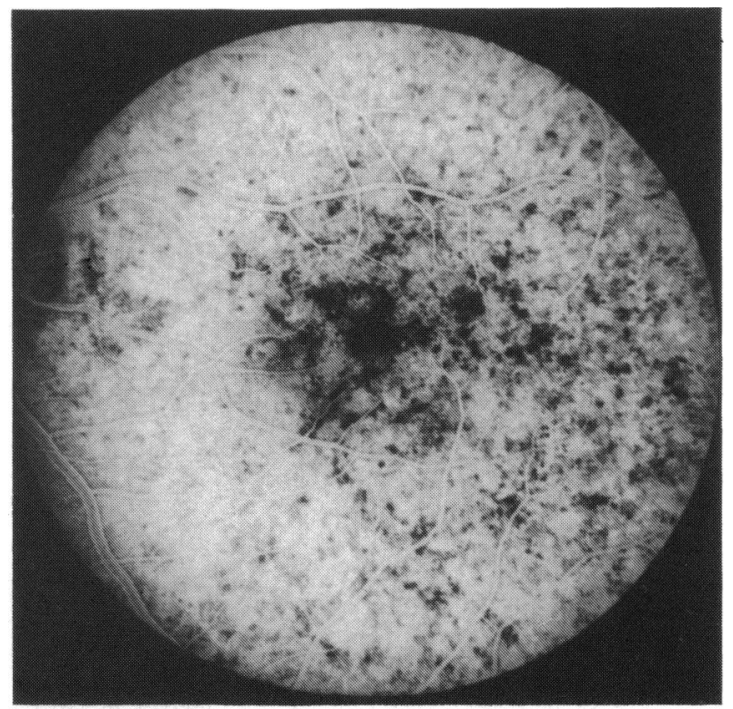

Fig. 5 Fluorescein angiography of the left eye, showing retinal pigment epithelial changes only.

There are considerable differences in the degree of retinal dysfunction in the reported cases of Bietti's disease, partly due no doubt to the progressive nature of the disorder, but as more cases are reported the parameters of clinical severity may become more evident. Long term follow-up of only two cases has been reported. ${ }^{2}$ The two brothers originally seen by Bietti were re-examined by Bagolini and Ioli-Spada 30 years later. Both had suffered a marked decline in central vision and a contraction of visual field. The corneal dystrophy was unchanged, but the chorioretinal degeneration had advanced, though the number of crystalline deposits had decreased. The discs were slightly pale, and the retinal vessels were narrowed.

There are insufficient cases of Bietti's disease to establish a pattern of inheritance, if any, but the presence of two sibships and the child of a consanguineous marriage among the 10 reported cases ${ }^{12}$ suggests an autosomal recessive disorder. Five cases of crystalline retinopathy similar to Bietti's but without the corneal dystrophy have been reported. ${ }^{11-13}$ The presence of sibships and consanguinity within this group also suggests an autosomal recessive disease, but at present we do not know whether these cases share a common aetiology with Bietti's disease or merely show a clinical similarity.

We thank Angela Hobley and Dr Charles Pennock for their assistance, Jill Bennerson for the photographs, and Gary James for the drawing (Fig. 1). 


\section{References}

1 Bietti GB. Su alcune forme atipiche o rare degenerazione retinica (degenerazione tappeto-refiniche e quadri morobisi similari). Boll Oculist 1937; 16: 1159-1244.

2 Bagolini B, Ioli-Spada G. Bietti's tapetoretinal degeneration with marginal corneal dystrophy. Am J Ophthalmol 1968; 65: 53-60.

3 Welch RB. Bietti's tapetoretinal degeneration with marginal corneal dystrophy. Crystalline retinopathy. Trans Am Ophthalmol Soc 1977; 75: 164-79.

4 Sanderson PO, Kuwabara T, Stark WJ, Wong VG, Collins EM. Cystinosis. A clinical, histopathologic, and ultrastructural study. Arch Ophthalmol 1974; 91: 270-4.

5 Fielder AR, Garner A, Chambers TL. Ophthalmic manifestations of primary oxalosis. Br J Ophthalmol 1980; 64: 782-8.

6 Bullock JD, Albert DM. Flecked retina appearance secondary to oxalate crystals from methoxyflurane anesthesia. Arch Ophthalmol 1975; 93: 26-31.
7 Takki K. Gyrate atrophy of choroid and retina associated with hyperornithinaemia. Br J Ophthalmol 1974; 58: 3-23.

8 Gilbert WR Jr, Smith JL, Nyhan WL. The Sjögren-Larssen syndrome. Arch Ophthalmol 1968; 80: 308-16.

9 Fraunfelder FT. Interim report. National registry of possible drug-induced ocular side effects. Ophthalmology (Rochester) 1980; 87: 87-90.

10 Craandijk A, Houtsmuller AJ. Hypo and abetalipoprotienaemia. In: Winkelman JE, Crone RA, eds. Perspectives in ophthalmology. Amsterdam: Exerpta Medica, 1970: 2: 96-102.

11 François J, De Laey JJ. Bietti's crystalline fundus dystrophy. Ann Ophthalmol 1978; 10: 709-16.

12 Grizzard WS, Deutman AF, Nijhuis F, Aam De Kerk A Crystalline retinopathy. Am J Ophthalmol 1978; 86: 81-8.

13 Maudlin WM, O'Connor PS. Crystalline retinopathy (Bietti's tapetoretinal degeneration without marginal corneal dystrophy). Am J Ophthalmol 1981; 92: 640-6.

Accepted for publication 2 May 1986. 